\title{
Inactivation of hMLH1 and $h M S H 2$ by promoter methylation in primary non-small cell lung tumors and matched sputum samples
}

\author{
Yi-Ching Wang, ${ }^{1}$ Yung-Pin Lu, ${ }^{1}$ Ruo-Chia Tseng, ${ }^{1}$ Ruo-Kai Lin, ${ }^{1}$ Jer-Wei Chang, ${ }^{1}$ \\ Jung-Ta Chen, ${ }^{2}$ Chuen-Ming Shih, ${ }^{3}$ and Chih-Yi Chen ${ }^{4}$
}

${ }^{1}$ Department of Biology, National Taiwan Normal University, Taipei, Taiwan, Republic of China
2Department of Pathology, Veterans General Hospital, Taichung, Taiwan, Republic of China
${ }^{3}$ Department of Internal Medicine, China Medical College Hospital, Taichung, Taiwan, Republic of China
${ }^{4}$ Department of Thoracic Surgery, Veterans General Hospital, Taichung, Taiwan, Republic of China

We performed a genetic and epigenetic study of the $b M L H 1$ and $b M S H 2$ mismatch repair genes in resected primary tumors from 77 non-small cell lung cancer (NSCLC) patients. The molecular alterations examined included the loss of mRNA and protein expression as well as promoter methylation, and the allelic imbalance of the chromosomal regions that harbor the genes. We found that $78 \%$ and $26 \%$ of patients showed at least one type of molecular alteration within the $b M L H 1$ and $b M S H 2$ genes, respectively. Promoter methylation of the $b M L H 1$ gene was present in $55.8 \%$ of tumors, and was significantly associated with the reduction in $\mathrm{mRNA}$ and protein expression $(P=0.001)$. A $72 \%$ concordance of aberrant methylation in sputum samples with matched resected tumors was found. In addition, a 93\% consistency between the promoter methylation and the mRNA expression of the $h M S H 2$ gene was found in 14 female NSCLC patients. However, no correlation was found between the expression of hMLH1 and hMSH2 proteins and the allelic imbalance of five microsatellite markers closely linked to the genes. Our results suggest that $b M L H 1$ is the major altered mismatch repair gene involved in NSCLC tumorigenesis, and that promoter methylation is the predominant mechanism in $b M L H 1$ and $b M S H 2$ deregulation. In addition, promoter methylation of the $b M L H 1$ gene may be identified in sputum samples to serve as a potential diagnostic marker of NSCLC.

J. Clin. Invest. 111:887-895 (2003). doi:10.1172/JCI200315475.

\section{Introduction}

Lung cancer is one of the most common malignancies in the world and is the leading cause of cancer deaths in industrial countries, including Taiwan (1). Recently, a marker for genetic instability, microsatellite instability (MSI), has been identified in a class of familial colon carcinomas known as hereditary nonpolyposis colorectal cancer $(2,3)$ and in a wide variety of other human cancers including lung cancer (4-8). It was reported that MSI was associated with mutations in mismatch repair genes such as $b M L H 1$ and $b M S H 2$ (2, $3)$ in hereditary nonpolyposis colorectal cancer. Somatic mutations and promoter hypermethylation of mismatch repair genes have also been shown in a

Received for publication March 18, 2002, and accepted in revised form January 28, 2003.

Address correspondence to: Yi-Ching Wang, Department of Biology, National Taiwan Normal University, No. 88, Section 4, Tingchou Road, Taipei 116, Taiwan, Republic of China. Phone: 886-2-2933-6876 ext. 373; Fax: 886-2-2931-2904; E-mail: t43017@cc.ntnu.edu.tw.

Conflict of interest: The authors have declared that no conflict of interest exists.

Nonstandard abbreviations used: microsatellite instability (MSI); non-small cell lung cancer (NSCLC); loss of heterozygosity (LOH); squamous cell carcinoma (SQ); adenocarcinoma (AD); methylation-specific PCR (MSP); 5-aza-2'-deoxycytidine (5-aza-dC). proportion of colon, gastric, and endometrial tumors with MSI (5-10). However, the data on mismatch repair gene alterations in lung cancer is scarce.

To examine the etiological association of genetic instability in lung tumorigenesis, we previously investigated the frequency of MSI as well as the association between MSI and expression of hMLH1 mismatch repair protein in 68 patients with non-small cell lung cancer (NSCLC) (11). Forty-one percent of the patients demonstrated instability in multiple tested microsatellite markers and $77 \%$ of MSI patients showed no expression of hMLH1 protein. In addition, hMLH1 protein expression was undetectable in $60.9 \%$ of NSCLCs. In support of our study, Xinarianos et al. (12) have also reported that $58.6 \%$ of NSCLC samples had reduced expression levels of the hMLH1 protein. However, they found no mutations in the promoter or exons of the $b M L H 1$ gene examined.

To further identify the molecular basis for loss of hMLH1 protein expression and to examine the possible involvement of another mismatch repair protein, hMSH2, in NSCLC tumorigenesis, we performed a comprehensive genetic and epigenetic study of protein and mRNA expression as well as promoter methylation and loss of heterozygosity (LOH) of the bMLH1 and $b M S H 2$ genes in 77 resected primary NSCLC samples, performed in parallel to the clinicopathological 
analyses of the patients. Our data indicate that promoter methylation is the predominant mechanism in immunohistochemical negativity of the $h M L H 1$ and $b M S H 2$ genes and that promoter methylation of the bMLH1 gene can be identified in sputum samples to serve as a potential diagnostic marker of NSCLC.

\section{Methods}

Study population, tumor samples, and sputum samples. The study subjects were 77 patients diagnosed with primary NSCLC admitted to Veterans General Hospital, Taichung, Taiwan, between 1993 and 2000. Forty-two of them had squamous cell carcinomas (SQs), 28 had adenocarcinomas (ADs), four had adenosquamous cell carcinomas, and three had large-cell carcinomas. Histological classification of the tumor types and stages was performed according to the World Health Organization classification method and the Tumor, Node, Metastasis System, respectively. Information on the smoking history of the lung cancer patients was obtained from hospital records. The study was reviewed and approved by the institution's Surveillance Committee, which allowed us to obtain tissue samples and all pertinent followup information.

Surgically resected tumor samples were immediately snap-frozen and were subsequently stored in liquid nitrogen. For the methylation assay, genomic DNA was prepared using proteinase $\mathrm{K}$ digestion and phenol/chloroform extraction, followed by ethanol precipitation. For $\mathrm{LOH}$ analysis, samples were microdissected in order to recover tumor tissue from up to five serial $5-\mu \mathrm{m}$ sections of paraffin-embedded tumor specimens. Following dewaxing in xylene, genomic DNA was extracted according to the standard methods described above.

Sputum samples were obtained from 29 NSCLC patients 3 months before surgery, following standardized procedures. In addition, sputum samples obtained from ten cancer-free individuals served as control during methylation assays. DNA was extracted from 50\% of the homogenized pellets of sputum using a tissue kit (QIAGEN GmbH, Hilden, Germany) according to the manufacturer's specifications.

Analysis of protein expression: immunohistochemistry assay. Blocks of paraffin-embedded tumors were cut into 5$\mu \mathrm{m}$ slices and then processed using protocols described previously (11). The tumors were analyzed for hMLH1 and hMSH2 protein expression by the immunohistochemistry assay. Monoclonal antibodies used were G168-728 (1:250; PharMingen, San Diego, California, USA) for the hMLH1 protein and FE11 (1:50; Oncogene Science, Cambridge, Massachusetts, USA) for the hMSH2 protein. The normal staining pattern for hMLH1 and hMSH2 is nuclear. Tumor cells that exhibited an absence of nuclear staining in the presence of non-neoplastic cells and infiltrating lymphocytes with nuclear staining were considered to have an abnormal pattern. Staining results were examined without knowledge of the status of the molecular analyses.
Analysis of $m R N A$ expression: RT-PCR assay. Total RNA was prepared from matched pairs of primary tumors and nearby normal lung tissues using TRIZOL reagent (Gibco; Invitrogen, Carlsbad, California, USA). SuperScript reverse transcriptase (Gibco; Invitrogen) was used to synthesize cDNA with protocols provided by the manufacturer.

Expression levels of the $b M L H 1$ and $b M S H 2$ genes were detected by a multiplex PCR assay using the $\beta$-actin gene as an internal control to indicate the amount of RNA in each reaction. The primer nucleotide sequences were as follows: for the $h M L H 1$ gene, sense, $5^{\prime}$ GTGCTGGCAATCAAGGGACCC-3', antisense, 5'-CACGGTTGAGGCATTGGGTAG-3'; for the $h M S H 2$ gene, sense, 5'-GTCGGCTTCGTGCGCTTCTTT- ${ }^{\prime}$ ', antisense, $5^{\prime}$-TCTCTGGCCATCAACTGCGGA-3'; for the $\beta$-actin gene, sense, 5'-ACACTGTGCCCATCTACGAGG-3' , antisense, 5'-AGGGGCCGGACTCGTCATACT- $3^{\prime}$. PCR was performed for 35 cycles with an annealing temperature of $66^{\circ} \mathrm{C}$ using cDNA synthesized from 50 ng total RNA.

To quantify the relative levels of mRNA expression in the multiplex RT-PCR assay, the value of the internal standard $(\beta$-actin $)$ in each reaction was used as the baseline gene expression of that sample and the relative value was calculated for the $b M L H 1$ and $b M S H 2$ genes for each tumor and matched normal samples. Tumor cells that exhibited a lower expression level than did normal cells were considered to have an abnormal pattern.

HpaII-based multiplex PCR methylation assay for the bMLH1 gene in tumor and sputum samples. The promoter methylation status of the $b M L H 1$ gene of 77 tumor samples and 29 sputum samples was investigated using HpaII-based multiplex PCR analysis. Genomic DNA (200 ng) was double digested either with $10 \mathrm{U}$ of methylation-sensitive enzyme (HpaII; New England Biolabs Inc., Beverly, Massachusetts, USA) or without enzyme for 10 hours at $37^{\circ} \mathrm{C}$. One hundred nanograms of the digested DNA was subjected to multiplex PCR. The primer nucleotide sequences for the $b M L H 1$ promoter region (-670 to $-67 \mathrm{bp}$ ) containing four HpaII sites used for analyses of resected tumor samples were as described (5). The internal control used for analyses was the IFN- $\beta 1$ sequence (sense, $5^{\prime}$-ATGAGCTACAACTTGCTTGGA-3', antisense, 5'-TCAGTTTCGGAGGTAACCTGT-3'), which contains no HpaII site. PCR was performed for 35 cycles at an annealing temperature of $64^{\circ} \mathrm{C}$. For sputum samples, a second PCR using 1:100 of the product of the first PCR was performed. Methylation was determined by the ratio of the normalized intensity ( $h M L H 1 /$ internal control) of the samples pretreated with HpaII to that of the undigested samples. DNA of normal lung tissues from 28 NSCLC patients and of sputum samples from ten cancer-free individuals was examined; ratios were less than 0.2 for all samples. Therefore, a ratio greater than 0.2 was defined as aberrant methylation.

Methylation-specific PCR assay for the bMLH1 and bMSH2 genes. The promoter methylation status of the $b M L H 1$ gene of all tumor samples and of the $b M S H 2$ 

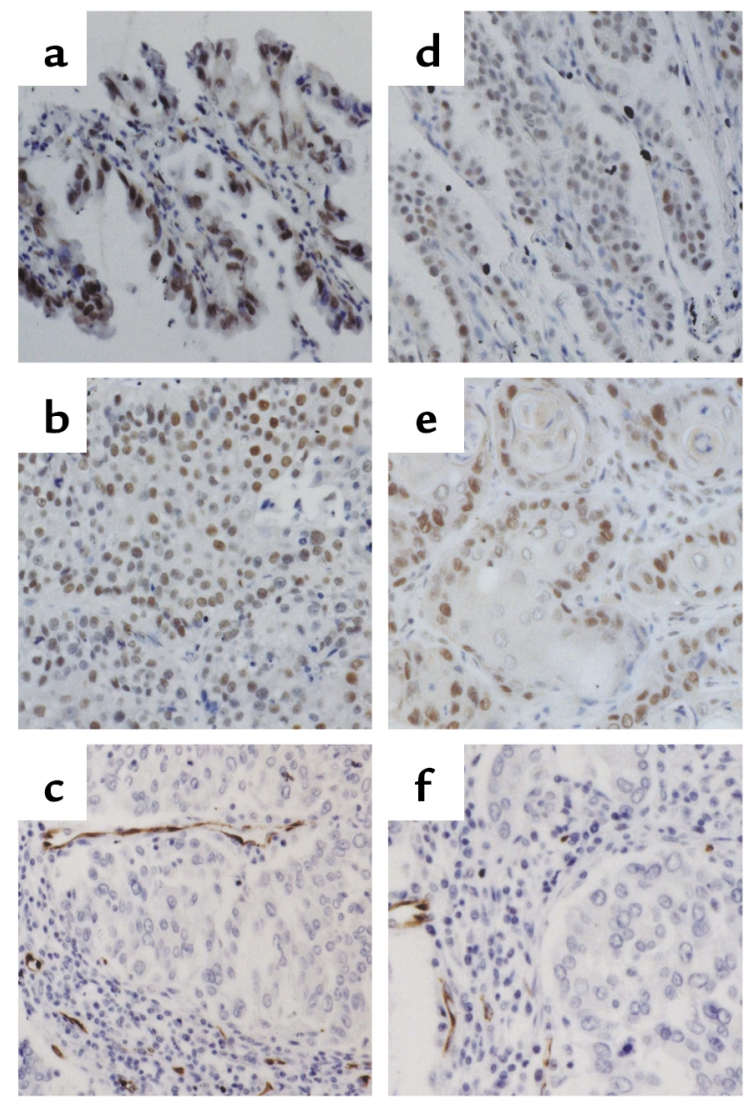

gene of tumor samples from 14 female lung cancer patients was determined by chemical treatment with sodium bisulfite and subsequent methylation-specific PCR (MSP) analysis as described (13). Primer sequences were as described (14). PCR was performed for 40 cycles with annealing temperatures of $65^{\circ} \mathrm{C}$ and $62^{\circ} \mathrm{C}$ for unmethylated and methylated reactions, respectively, using $50 \mathrm{ng}$ bisulfite-modified DNA. All PCRs were performed with positive controls for both unmethylated and methylated alleles and no DNA control.

Treatment of lung cancer cells with 5-aza-2'-deoxycytidine. The human lung cancer cell lines CL2 and CL15-F4 were derived from Taiwanese patients with $\mathrm{AD}$ of the lung (kindly provided by P.-C. Yang, Department of Internal Medicine, National Taiwan University Hospital, Taipei, Taiwan). Cells were plated at $10^{5}$ per $100-\mathrm{mm}$ culture dish on the day before treatment. The cultures were treated for three doubling times with $1 \mu \mathrm{M} 5$-aza-2'-deoxycytidine (5-aza-dC). The 5-aza-dC-containing medium was changed after each cell doubling during the treatment. On the day after the third doubling, cells were harvested for analysis of their methylation status using an MSP assay and analysis of mRNA production by an RT-PCR assay. In experiments for protein analysis, the cells were given fresh media without 5 -aza-dC for two more days and then fixed and stained for protein expression as judged by immunohistochemistry.

\section{Figure 1}

Immunohistochemical staining of hMLH1 protein expression (a-c) and hMSH2 protein expression ( $\mathbf{d}-\mathbf{f})$ in paraffin-embedded sections of lung tumor specimens. hMLH1 nuclear immunoreactivity was found in $\mathbf{a}$ and $\mathbf{b}$. hMSH2 nuclear immunoreactivity was found in $\mathbf{d}$ and $\mathbf{e}$. Tumors negative for hMLH1 1 and $\mathrm{hMSH} 2$ are shown in $\mathbf{c}$ and f, respectively. Original magnification $\times 100$.

LOH at chromosome regions $3 p 21.3-22$ and $2 p 16.3-22$ in microdissected tumor samples. Five microsatellite polymorphic markers for the $b M L H 1$ and $b M S H 2$ genes were used for LOH analysis: D3S3718 (3p21.33; 0.9 cM distal to the $b M L H 1$ locus), D3S1768 (3p21.3-22; $2.5 \mathrm{cM}$ distal to the $h M L H 1$ locus), D3S1612 (3p21.322; $2.5 \mathrm{cM}$ distal to the $b M L H 1$ locus), D2S123 (2p16.3; $3.6 \mathrm{cM}$ proximal to the $h M S H 2$ locus), and D2S1788 (2p22.3, $11.5 \mathrm{cM}$ distal to the $h M S H 2$ locus). Twenty nanograms of genomic DNA from normal lung tissues or microdissected tumor samples of 77 patients was used for PCR-based LOH analysis. The primer sequence for each microsatellite marker was obtained from the National Center for Biotechnology Information database. The allelic ratio was calculated as (T1/T2):(N1/N2), the ratio of tumor (T) alleles to normal $(\mathrm{N})$ alleles. $\mathrm{LOH}$ was defined as an allelic ratio above 2.0 or below 0.5 .

Analysis of MSI. MSI was analyzed in 21 sputum DNA samples using four microsatellite markers: D3S1234 (3p14), D9S162 (9p22-23), D13S170 (13q22-31), and D17S786 (17p13.1). These markers were selected because they were highly sensitive at detecting MSI in our previous study (11). PCR conditions were as described (11). MSI was revealed by the presence of expansions or contractions of repeats in sputum DNA that were absent from normal DNA from lung tissue of the same patient. An MSI-positive result was defined as one demonstrating instability in one or more markers.

Statistical analysis. The Pearson $\chi^{2}$ test was used to compare the frequency of $b M L H 1$ and $b M S H 2$ alterations between NSCLC patients with different characteristics, including sex and smoking status, and various clinicopathological parameters, such as tumor type and tumor stage, respectively. Comparison of age between patients with and without the alteration was made by the two-sample $t$ test. The

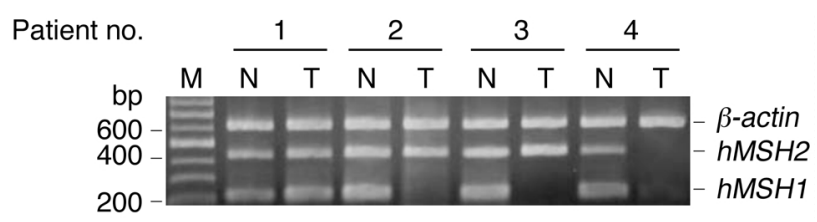

\section{Figure 2}

Representative examples of multiplex RT-PCR analysis of the $h M L H 1$ and $h M S H 2$ genes in lung cancer patients. Amplification of the $\beta$-actin gene indicated the initial RNA template used for each reaction. Patient numbers are the sample numbers. $\mathrm{N}$ and $\mathrm{T}$ represent the paired normal and tumor lung cells from the same patient. $\mathrm{M}$, molecular marker. 
Table 1

Genetic alterations of the $h M L H 1$ gene in relation to the clinicopathological parameters of resected NSCLC tumors

\begin{tabular}{|c|c|c|c|c|c|c|c|c|c|}
\hline \multirow[b]{2}{*}{ Characteristic } & \multicolumn{3}{|c|}{ Protein expression } & \multicolumn{3}{|c|}{ mRNA expression } & \multicolumn{3}{|c|}{ Promoter methylation } \\
\hline & Total & + & $-^{A}(\%)$ & Total & + & $-{ }^{A}(\%)$ & Total & - & $+^{A}(\%)$ \\
\hline All patients ${ }^{\mathrm{B}}$ & 77 & 37 & $40(51.9)$ & 77 & 34 & $43(55.8)$ & 77 & 34 & $43(55.8)$ \\
\hline Age $($ mean $\pm S D)$ & $64 \pm 10$ & $64 \pm 13$ & $66 \pm 9$ & $63 \pm 13$ & $64 \pm 10$ & $64 \pm 13$ & & & \\
\hline \multicolumn{10}{|l|}{ Sex } \\
\hline Male & 63 & 32 & $31(49.2)$ & 63 & 29 & $34(56.2)$ & 63 & 29 & $34(54.0)$ \\
\hline Female & 14 & 5 & $9(64.3)$ & 14 & 5 & $9(64.3)$ & 14 & 5 & $9(64.3)$ \\
\hline \multicolumn{10}{|l|}{ Smoking history } \\
\hline Smoker & 57 & 31 & $26(45.6)^{c}$ & 57 & 27 & $30(52.6)$ & 57 & 27 & $30(52.6)$ \\
\hline Nonsmoker & 20 & 6 & $14(70.0)$ & 20 & 7 & $13(65.0)$ & 20 & 7 & $13(65.0)$ \\
\hline \multicolumn{10}{|l|}{ Tumor type } \\
\hline SQ & 42 & 23 & $19(45.2)$ & 42 & 23 & $19(45.2)^{\mathrm{D}}$ & 42 & 21 & $21(50.0)$ \\
\hline$A D$ & 28 & 10 & $18(64.3)$ & 28 & 8 & $20(71.4)$ & 28 & 11 & $17(60.7)$ \\
\hline \multicolumn{10}{|l|}{ Tumor stage } \\
\hline$I+I I$ & 40 & 14 & $26(65.0)$ & 40 & 20 & $20(50.0)$ & 40 & 18 & $22(55.0)$ \\
\hline$I I I+I V$ & 35 & 21 & $14(40.0)$ & 35 & 12 & $23(65.7)$ & 35 & 16 & $19(54.3)$ \\
\hline
\end{tabular}

AThese groups represent patients with alteration in the $h M L H 1$ gene/protein. ${ }^{B}$ Total number of samples in some categories is less than the overall number analyzed because the clinical data were not available for some samples. ${ }^{C} P=0.060$ by Pearson $\chi^{2}$ test. ${ }^{D} P=0.031$ by Pearson $\chi^{2}$ test.

Pearson $\chi^{2}$ test was also used for comparative analysis of expression of $h M L H 1$ and $b M S H 2$ proteins with allelic imbalance data.

\section{Results}

Protein and $m R N A$ expression of $h M L H 1$. We investigated hMLH1 protein expression using immunohistochemical analysis (Figure 1, a-c), and mRNA expression using RT-PCR analysis (Figure 2) in tumors from 77 primary NSCLC patients. The association of altered protein and mRNA expression with the clinicopathological parameters of patients was also examined (Table 1). Forty specimens (51.9\%) exhibited altered protein expression, and 43 specimens (55.8\%) showed an alteration of mRNA expression. A reduction in hMLH1 protein expression was found more frequently in tumors from nonsmoking patients than in those from smoking patients $(P=0.06)$. In addition, the lack of mRNA expression was significantly more prevalent in $\mathrm{AD}$ patients $(71.4 \%, 20$ of 28$)$ than in SQ patients $(45.2 \%, 19$ of 42$)(P=0.031)$. Promoter methylation of $h M L H 1$. We examined the methylation status of the $h M L H 1$ gene in 77 resected tumors using HpaII-based PCR methylation analysis and an MSP assay. Representative examples of HpaIIbased PCR methylation analysis are shown in Figure 3 b. Figure 3 a shows virtually no methylation in the $b M L H 1$ promoter of 28 normal lung tissues examined. Methylation of the $b M L H 1$ gene was present in 43 of the 77 (55.8\%) tumors (Table 1). A 74\% concordance of the methylation status for the $b M L H 1$ gene was found between the HpaII-based PCR and the MSP assays (Figure 3d).

Aberrant promoter methylation of the $b M L H 1$ gene was found in $55 \%$ of stage I and II patients, suggesting that it is an early and very frequent event in NSCLC.
We then examined whether aberrant methylation might also be found in the sputum of patients with NSCLC and whether aberrant methylation can track the cancer cell even in patients for which conventional sputum cytology is not sensitive enough. Cytologically negative sputum samples were available from 29 NSCLC patients and were analyzed for $h M L H 1$ methylation status (Figure 3c). HpaII-based PCR analysis showed aberrant methylation in 11 of the 29 (37.9\%) sputum samples. In addition, a $72 \%$ (21 of 29$)$ concordance of sputum samples with matched resected tumors was found (Table 2).

To further compare the sensitivity and specificity of promoter methylation and MSI in detecting NSCLC in sputum samples, 21 sputum samples were also examined for MSI. Sensitivity was $60 \%$ for methylation analysis, but only $22 \%$ for MSI analysis (Table 2).

Protein and $m R N A$ expression of hMSH2. We investigated expression of hMSH2 protein (Figure 1, d-f) and mRNA (Figure 2) in tumors from 77 primary NSCLC patients. The association of altered protein and mRNA expression with the clinicopathological parameters of patients was also examined (Table 3 ). Fourteen specimens (18.2\%) and thirteen specimens (16.9\%) exhibited an alteration of protein and mRNA expression, respectively. Negative hMSH2 protein expression was significantly associated with younger patients $(P=0.021)$, female patients $(42.9 \%, P=0.005)$, and nonsmokers $(40.0 \%, P=0.003)$ (Table 3 ). In addition, the number of mRNA-negative samples was significantly higher in $\mathrm{AD}$ patients $(28.6 \%, 8$ of 28$)$ than in SQ patients $(9.5 \%, 4$ of 42$)(P=0.038)$.

Promoter methylation of hMSH2. We examined the methylation status of the $\mathrm{bMSH} 2$ gene in resected tumors from 14 female lung cancer patients using the 


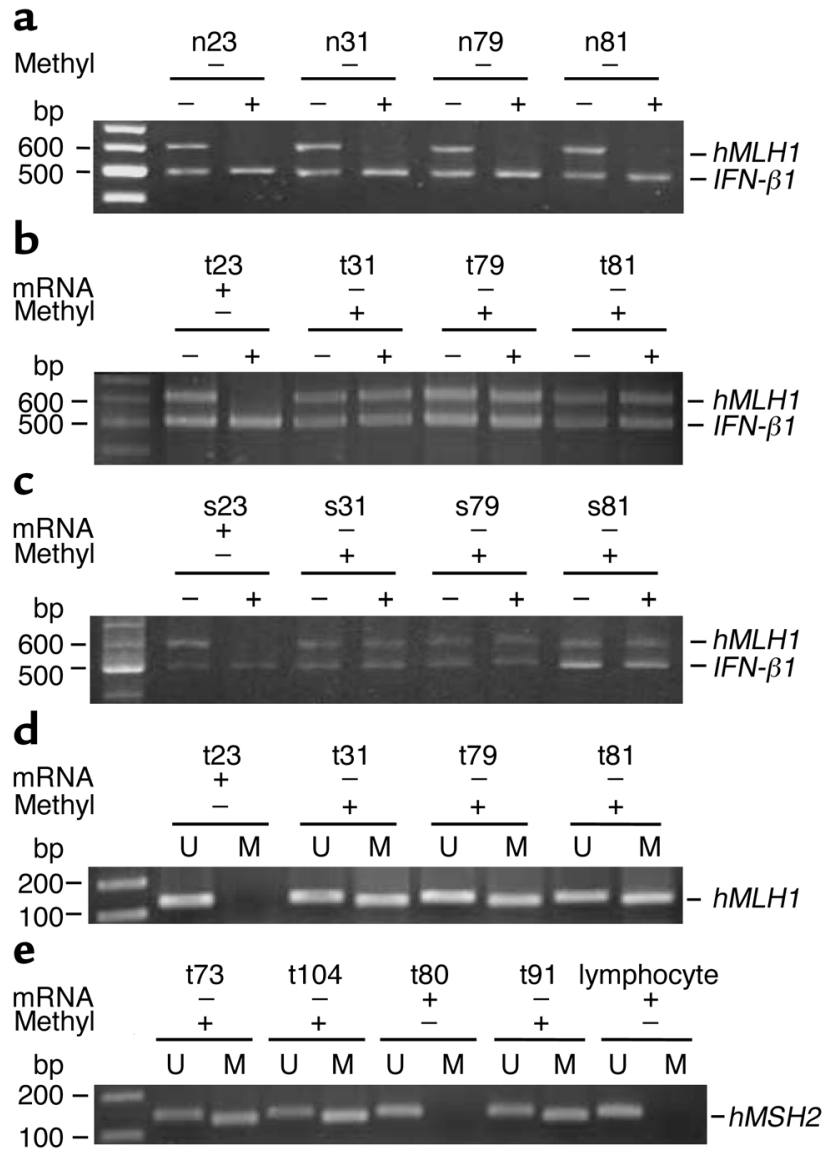

MSP assay because reduction in hMSH2 expression appeared to be more prevalent in female patients (Table 3). Representative examples of the MSP analysis are shown in Figure 3e. Methylation of the hMSH2 gene was present in four (28.6\%) tumors from female lung cancer patients. These four tumors all lacked hMSH 2 mRNA expression. In addition, a 93\% (13 of 14) concordance in mRNA expression and promoter methylation patterns was found.

Correlation of promoter methylation with negative $m R N A$ and protein expression, and reactivation of $h M L H 1$ and bMSH2 by 5-aza-dC treatment. The data for protein expression, mRNA expression, and promoter methylation were then cross-examined to investigate the correlation among these three parameters for the bMLH1 gene (Figure 4a). The results indicated that negative protein expression was significantly associated with negative mRNA expression and promoter methylation of the $b M L H 1$ gene $(P=0.001)$. The concordant group was found in $68.9-71.5 \%$ of the patients analyzed. In addition, negative protein expression was significantly associated with negative hMSH2 mRNA

Table 2

\section{Figure 3}

Representative examples of promoter methylation analysis of the hMLH1 (a-d) and hMSH2 (e) genes. (a-c) Hpall-based multiplex PCR assay for the $h M L H 1$ promoter. (a) Normal lung tissues, (b) tumor lung samples, and (c) matched sputum samples, indicated by $\mathrm{n}, \mathrm{t}$, and $\mathrm{s}$ before subject identification number. +, Hpall digest; -, mock digest. Positions of the target $h M L H 1$ gene and control IFN- $\beta 1$ gene are indicated. Normal lung tissues showed no $h M L H 1$ promoter methylation. MSP assay for the $h M L H 1$ promoter (d) and (e) $h M S H 2$ promoter. Primer sets used for amplification are designated $U$ for unmethylated or $\mathrm{M}$ for methylated genes. The data for lymphocytes containing unmethylated $h M S H 2$ are shown for the experimental control. + and - in the rows labeled methyl and mRNA represent the status of promoter methylation and mRNA expression.

expression (Figure $4 \mathrm{~b} ; P=0.002$ ). However, there was no correlation between altered expression of $b M S H 2$ and $h M L H 1$ (data not shown).

To determine whether $b M L H 1$ and $b M S H 2$ promoter methylation could be further linked to the loss of gene expression, two lung cancer cells that showed negative expression and promoter hypermethylation of the $b M L H 1$ or $b M S H 2$ gene were treated with the demethylating agent 5-aza-dC. As shown in Figure 5, treatment with 5-aza-dC successfully restored mRNA and protein expression and demethylated the promoter region in each of the cells that lacked $b M L H 1$ or $b M S H 2$ expression and that harbored a methylated respective promoter.

No correlation of allelic imbalance at 3p21.3-22 and 2p16.3-22 with hMLH1 and bMSH2 protein expression. Microdissected genomic DNA from tumors and matched normal lung tissue was examined for the incidence of allelic imbalance at chromosome regions 3p21.3-22 and 2p16.3-22, where bMLH1 and bMSH2 reside, using five microsatellite markers. The frequencies of $\mathrm{LOH}$ were $15.8-37.5 \%$ and $9.3-36.4 \%$ for the regions at $3 \mathrm{p} 21.3-22$ and $2 \mathrm{p} 16.3-22$, respectively. The patients with $\mathrm{LOH}$ at these regions were mostly SQ patients (data not shown). Statistical analysis showed close correlation between the $\mathrm{LOH}$ of markers nearby, suggesting that $\mathrm{LOH}$ at these markers represents the

Comparison of $h M L H 1$ promoter methylation and MSI in matched sputum and tumor DNA from NSCLC patients $h M L H 1$ promoter methylation

Class

$\mathrm{S}+/ \mathrm{T}+$

$\mathrm{S}-/ \mathrm{T}-$

$\mathrm{S}-/ \mathrm{T}+$

$\mathrm{S}+/ \mathrm{T}-$

Total samples
Patient no. Sensitivity Specificity

$9(31 \%)$

$12(41 \%)$

$6(21 \%)$

$2(7 \%)$

29
MSI

Patient no. Sensitivity Specificity $2(10 \%) \quad 22 \% \quad 100 \%$ $12(57 \%)$

$7(33 \%)$

$0(0 \%)$

21
S, sputum; T, tumor; +, with alteration; -, without alteration. Sensitivity is defined as the ratio of correctly identified positive sputum samples ("true positives") in all existing tumor-positive patients ("true positive" plus "false negative"). Specificity is defined as the ratio between correctly identified cases with no molecular alteration ("true negative") and all existing alteration-free tumor samples ("true negative" plus "false positive"). The total number of samples in the analysis of MSI is 21 , because 8 samples had no sputum DNA available for analysis. 
Table 3

Genetic alterations of the $h M S H 2$ gene in relation to the clinicopathological parameters of resected NSCLC tumors

\begin{tabular}{|c|c|c|c|c|c|c|}
\hline \multirow[b]{2}{*}{ Characteristics } & \multicolumn{3}{|c|}{ Protein expression } & \multicolumn{3}{|c|}{ mRNA expression } \\
\hline & Total & + & ${ }^{\mathrm{A}}(\%)$ & Total & + & ${ }^{\mathrm{A}}(\%)$ \\
\hline All patients ${ }^{B}$ & 77 & 63 & $14(18.2)$ & 77 & 65 & $13(16.9)$ \\
\hline Age $($ mean $\pm S D)$ & $66 \pm 11$ & $57 \pm 13^{C}$ & $65 \pm 9$ & $64 \pm 12$ & & \\
\hline \multicolumn{7}{|l|}{ Sex } \\
\hline Male & 63 & 55 & $8(12.7)^{\mathrm{D}}$ & 63 & 54 & $9(14.3)$ \\
\hline Female & 14 & 8 & $6(42.9)$ & 14 & 10 & $4(28.6)$ \\
\hline \multicolumn{7}{|l|}{ Smoking history } \\
\hline Smoker & 57 & 51 & $6(10.5)^{\mathrm{E}}$ & 57 & 52 & $8(14.0)$ \\
\hline Nonsmoker & 20 & 12 & $8(40.0)$ & 20 & 15 & $5(25.0)$ \\
\hline \multicolumn{7}{|l|}{ Tumor type } \\
\hline SQ & 42 & 36 & $6(14.3)$ & 42 & 38 & $4(9.5)^{F}$ \\
\hline$A D$ & 28 & 22 & $6(21.4)$ & 28 & 20 & $8(28.6)$ \\
\hline \multicolumn{7}{|l|}{ Tumor stage } \\
\hline $1+I I$ & 40 & 33 & $7(17.5)$ & 40 & 33 & $7(17.5)$ \\
\hline$I I I+I V$ & 35 & 29 & $6(17.1)$ & 35 & 29 & $6(17.1)$ \\
\hline
\end{tabular}

AThese groups represent patients with alteration in the $h M S H 2$ gene/protein. ${ }^{B}$ Total number of samples in some categories is less than the overall number analyzed because clinical data were not available for some samples. ${ }^{C} P=0.021$ by two-sample $t$ test. ${ }^{D} P=0.005$ by Pearson $\chi^{2}$ test. E $P=0.003$ by Pearson $\chi^{2}$ test. ${ }^{\mathrm{F}} P=0.038$ by Pearson $\chi^{2}$ test.

frequency of allelic imbalance in the region where they are located (data not shown). However, comparative analysis of allelic imbalance data of these five markers with expression of hMLH1 and hMSH2 protein showed no correlation (Table 4; $P>0.05$ ).

\section{Discussion}

Aberrant methylation, which can result in the transcriptional silencing of the target gene, was frequently found in the $h M L H 1$ and $h M S H 2$ alterations in NSCLC tumors. A highly significant correlation was found between $b M L H 1$ gene methylation and negative protein and mRNA expression $(P=0.001)$, and a $93 \%$ concordance of bMSH2 methylation and mRNA expression patterns was shown in tumors from female lung cancer patients, suggesting that promoter methylation is the predominant mechanism by which these two genes are silenced in NSCLC. This conclusion is further strengthened by the re-expression of mRNA and protein, together with demethylation at the promoter region in both the $b M L H 1$ and $b M S H 2$ genes, after 5 -aza-dC treatment of two lung cancer cell lines. In addition, the frequent occurrence of promoter methylation of the $b M L H 1$ gene in early-stage tumors and sputum samples indicates its potential use as a diagnostic marker in NSCLC.

In this study, both HpaII and MSP assays were used to examine the methylation status of the $h M L H 1$ gene. HpaII-based PCR analysis examines methylation status at the region from -670 to -67 bp relative to the transcription start site of the $h M L H 1$ gene. This region contains four $\mathrm{HpaII}$ sites at $-567,-527,-374$, and $-341 \mathrm{bp}$. MSP analysis examines the methylation status at region -721 to $-598 \mathrm{bp}$. The primer design can detect a total of seven CpG sites at $-715,-709$, $-695,-693,-621,-619$, and $-609 \mathrm{bp}$. The methylation frequencies detected by the HpaII-based PCR and MSP assays were $55.8 \%$ and $37.7 \%$, respectively. A $74 \%$ concordance of the methylation status for the $b M L H 1$ gene between the two assays was observed. There seem to be at least three possible explanations for the $26 \%$ discordance between the two assays. First, the lower methylation frequency detected by the MSP assay compared with the HpaII-based assay may partly result from the fact that the MSP method was designed to analyze more CPG sites than was the HpaII method. Second, the regions examined in the two assays were different, and the methylation frequency of the various $\mathrm{CPG}$ sites may have been different. Finally, there may have been several distinct subpopulations present in the discordant tumors, subpopulations that had a mixture of cells with methylated and nonmethylated DNA or that each had methylation at different sites. It has been shown that the presence of promoter hypermethylation for a given gene can be heterogeneous at different $\mathrm{CPG}$ sites and/or with various tumor samples $(15,16)$. Several potential transcription factor binding sites, such as the Sp1, CCAAT-box, $\mathrm{C} / \mathrm{EBP}$, and $\mathrm{c}-\mathrm{Myb}$ binding sites, are located in the region from -552 to $-132 \mathrm{bp}(5,17,18)$. In addition, the methylation status of the promoter region examined by the HpaII assay is better correlated to the transcriptional activity of the $b M L H 1$ gene than is the MSP assay.

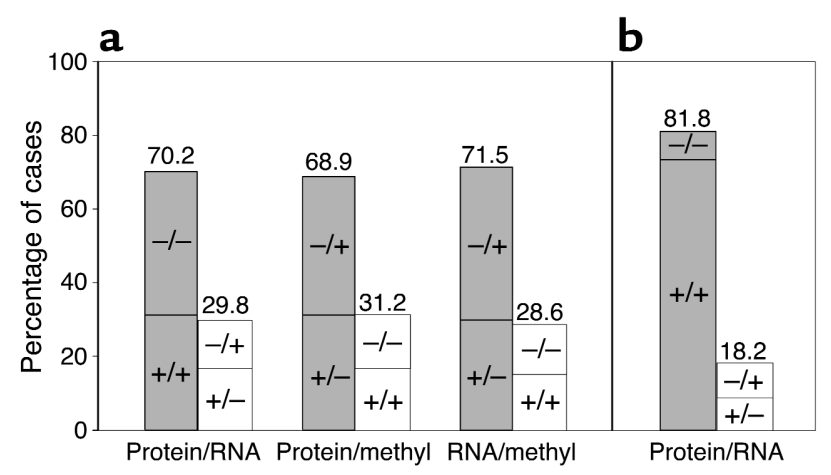

\section{Figure 4}

Concordance analysis between protein expression, mRNA expression, and promoter methylation of $h M L H 1$ (a) and $h M S H 2(\mathbf{b})$. The percentage of cases is indicated on the $y$ axis, whereas the type of comparison is shown on the $x$ axis. + indicates positive protein expression (protein), positive mRNA expression (RNA), and hypermethylation (methyl) of the promoter. Numbers above bars indicate percentages of the total concordant group (gray sections) and nonconcordant group (white sections). $P=0.001$ for association between protein expression, mRNA expression, and promoter methylation in the $h M L H 1$ gene; $P=0.002$ for association between protein expression and mRNA expression in the $h M S H 2$ gene. 
a

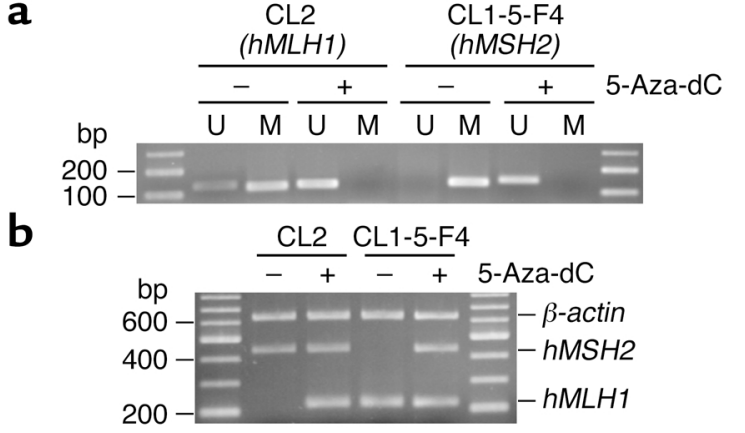

C $\mathrm{CL} 2-\mathrm{Aza}(\mathrm{hMLH1})$
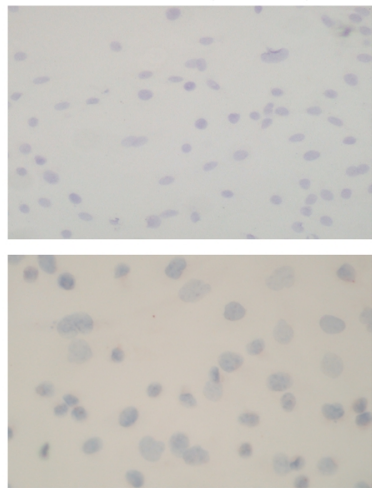

CL1-5-F4 - Aza (hMSH2)

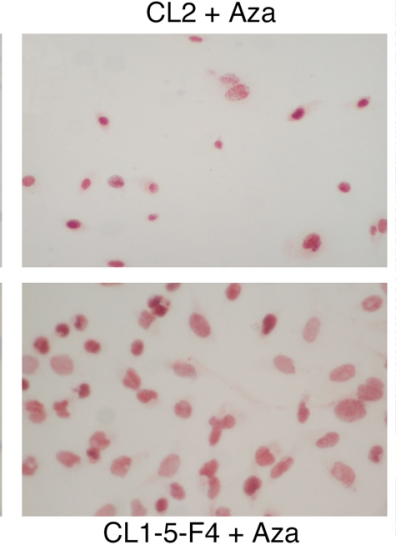

therefore, we used the HpaII result for all analyses, including correlation with clinicopathological parameters and gene expression. To ensure the reliability of the HpaII methylation assay, efforts were made to avoid incomplete digestion and false amplification using strict quality-control procedures. We consider technique artifacts to be an unlikely explanation for the discordance between the two assays. It will be important to examine the methylation status of all $\mathrm{CPG}$ sites within a much larger region to more precisely define the site critical for gene silencing in the future.

It has been reported that promoter methylation can be a very early event in NSCLC tumorigenesis $(19,20)$. This is consistent with our results in that bMLH1 methylation was detected in $55 \%$ of stage I and stage II tumors (Table 1) and in $37.9 \%$ (11 of 29) of the cytologically negative sputum samples. In addition, a $72 \%$ (21 of 29) concordance of $b M L H 1$ methylation status

\section{Figure 5}

Effects of 5-aza-dC treatment on CL2 and CL1-5-F4 cells. (a) Demethylation analysis by MSP assay of $h M L H 1$ and $h M S H 2$ in lung cancer cell lines after 5 -aza-dC treatment. The presence of the unmethylated product $(U)$ after 5 -aza-dC treatment indicates demethylation of the promoter in these cell lines. (b) RT-PCR analysis of mRNA expression of the $h M L H 1$ and $h M S H 2$ genes in cell lines. (c) Immunohistochemistry analysis of hMLH1 (original magnification $\times 100$ ) and $\mathrm{hMSH} 2$ (original magnification $\times 200$ ) protein expression in cell lines.

was detected in resected tumors and matched sputum samples (Figure 3). Sensitivity and specificity for methylation analysis in sputum DNA were $60 \%$ and $86 \%$, respectively (Table 2 ). To our knowledge, this is the first report of the potential use of promoter methylation of the $h M L H 1$ gene in sputum samples for the diagnosis of NSCLC, and is also the first report on the comparison of detection sensitivity between promoter methylation and MSI in sputum. The data from the $b M L H 1$ methylation analysis were consistent with the data reported previously for the detection of aberrant p16 $6^{\text {INK4a }}$ methylation (100\% sensitivity) and $\mathrm{O}^{6}$-methylguanine DNA methyltransferase gene methylation (67\%) in sputum DNA (21). Only two studies to date have reported the presence of MSI in the sputum of two SCLC patients (22) and in sputum from three of five lung cancer patients (23). Our data showed better sensitivity by promoter methylation than by MSI in detecting cancer in sputum DNA. Ahrendt et al. (24) have also reported p16 methylation to be more sensitive than MSI in detecting cancer in bronchoalveolar lavage samples. It was estimated that the detection limit can be as sensitive as one methylation allele in 1,000-50,000 unmethylated alleles using the two-stage PCR approach for $b M L H 1$ promoter methylation as we did $(17,21)$. However, the detection limit is only 1 in 200-500 for MSI analysis (22). Studies of DNA methylation in lung cancer and matched sputum samples to date strongly suggest that the analysis of DNA methylation patterns could become a powerful tool for accurate and early lung cancer diagnosis, with reasonable specificity and sensitivity. Our findings suggest that hypermethylation changes in multiple genes in sputum DNA might detect a very high-risk status or the

\section{Table 4}

Expression levels of hMLH1 and hMSH2 in NSCLC patients in relation to allelic imbalance at chromosomes 3p21.3-22 and 2p16.3-22

\begin{tabular}{|c|c|c|c|c|c|c|c|c|c|c|c|}
\hline \multirow[b]{3}{*}{ Protein } & \multirow{3}{*}{$\begin{array}{c}\text { Expression } \\
\text { level }\end{array}$} & \multicolumn{6}{|c|}{$3 p 21.3-22$} & \multicolumn{4}{|c|}{$2 \mathrm{p} 16.3-22$} \\
\hline & & \multicolumn{2}{|c|}{ D3S3718 } & \multicolumn{2}{|c|}{ D3S1768 } & \multicolumn{2}{|c|}{ D3S1612 } & \multicolumn{2}{|c|}{ D2S123 } & \multicolumn{2}{|c|}{ D2S1788 } \\
\hline & & $\mathrm{H}$ & $\mathrm{L}$ & $\mathrm{H}$ & $\mathrm{L}$ & $\mathrm{H}$ & $\mathrm{L}$ & $\mathrm{H}$ & $\mathrm{L}$ & $\mathrm{H}$ & $\mathrm{L}$ \\
\hline \multirow[t]{2}{*}{ hMLH1 } & Normal & 18 & 6 & 7 & 5 & 19 & 6 & 22 & 4 & 9 & 4 \\
\hline & Reduced & 19 & 4 & 13 & 7 & 24 & 2 & 27 & 1 & 12 & 8 \\
\hline \multirow[t]{2}{*}{ hMSH2 } & Normal & 33 & 9 & \multicolumn{2}{|c|}{ ND } & 37 & 7 & 43 & 5 & \multirow{2}{*}{\multicolumn{2}{|c|}{ ND }} \\
\hline & Reduced & 4 & 1 & & & 6 & 1 & 6 & 0 & & \\
\hline
\end{tabular}

Only informative cases (heterozygous size of the two alleles in normal) were included in analysis. Statistical analysis indicated a low correlation between expression level of hMLH1 and hMSH2 to the allelic imbalance at all markers $\left(P>0.05\right.$ by Pearson $\chi^{2}$ test). H, heterozygous; L, LOH. LOH frequency: D3S3718, 21.3\%; D3S1768, 37.5\%; D3S1612, 15.7\%; D2S123, 9.3\%; D2S1788, 36.4\%. ND, not determined. 
actual presence of cancer and may be used as a sensitive and reliable molecular diagnostic method. This should be confirmed in larger patient subsets.

The observation of association of altered $b M L H 1$ and $b M S H 2 \mathrm{mRNA} /$ protein expression with nonsmoking $\mathrm{AD}$ patients is intriguing. Registry data indicate that only a few female lung cancer patients smoke cigarettes in Taiwan (25). In addition, most cases of lung cancer in nonsmoking women are $\mathrm{AD}$, which shows less correlation with smoking $(1,25)$. It is possible that geographical and/or ethnic factors account for frequent $h M L H 1$ and $b M S H 2$ alterations in Taiwanese NSCLC patients. In addition, environmental factors other than cigarette smoke and/or endogenous factors may be involved in the observed association of $h M L H 1$ and $b M S H 2$ alterations with nonsmoking $\mathrm{AD}$. These possibilities may be attributable to the discrepancy between the present study and a USA study that found no promoter methylation of the $b M L H 1$ gene in 20 NSCLC tumors (26). In addition, the varying results may be due in part to differences in the type and/or stage of tumors analyzed in the contrasting studies. For example, a study analyzing the protein expression levels of $h M L H 1$ and bMSH2 in 33 bronchioloalveolar carcinomas showed no detectable alteration in these proteins (27). Bronchioloalveolar carcinoma is a subtype of lung $\mathrm{AD}$ with distinct clinical and pathologic features (28).

We observed that the allelic imbalance at chromosome regions $3 \mathrm{p} 21.3-22$ and $2 \mathrm{p} 16.3-22$, which harbor the $b M L H 1$ and $h M S H 2$ genes, respectively, was not associated with altered expression of hMLH1 and hMSH2 protein (Table 4). Our data on $b M S H 2$ is in agreement with previous studies that showed low LOH frequency $(11-15 \%)$ at $2 \mathrm{p} 16.3-22(29,30)$ and no association of allelic imbalance of chromosome $2 \mathrm{p}$ with negative hMSH2 protein expression (12). However, Xinarianos et al. (12) found that reduced expression of hMLH1 protein correlated with allelic imbalance at chromosome $3 \mathrm{p} 21$ based on the study of a marker (D3S1289) located $17.1 \mathrm{cM}$ from the $h M L H 1$ locus. The investigators concluded that allelic loss seems to be a major genetic event involved in $b M L H 1$ silencing. However, $b M L H 1$ promoter methylation was not performed in their study. The data to date, including our study, suggest that chromosome $3 p$, especially $3 \mathrm{p} 12$, $3 \mathrm{p} 14.2$, and $3 \mathrm{p} 21.3$, represents highly unstable regions that undergo frequent allele loss associated with lung cancer of SQ and with smoking exposure $(12,29,31,32)$. However, no homozygous deletions or rearrangements in the $h M L H 1$ gene have been found to be associated with lung cancer so far $(19,29,33)$. A gene dosage effect on the stoichiometry and the activity of the mismatch repair complex has been proposed to explain the observed high frequency of $\mathrm{LOH}$ at the chromosome region harboring the $b M L H 1$ gene (29). However, the 3p21.3 region contains the most common known fragile site in the genome (31), and the frequency of discontinuous
LOH reported in various studies makes the possibility of mitotic recombination highly likely (31). Whether the frequent hemizygous 3p21.3 allelic deletions reflect only the fragility of this region with a predisposition to recombination events induced after smoking exposure or whether they also indicate the presence of an underlying tumor suppressor gene (or genes), perhaps resulting in tumorigenesis because of haploinsufficiency, merits further clarification.

Although mutational analysis was not performed on our samples, several studies have shown that no mutation was identified in the promoter region or hot-spot exons of the $h M L H 1(19,29)$ and $b M S H 2$ (29) genes in NSCLC samples. This suggests that mutations are unlikely to be a major cause of $h M L H 1 / b M S H 2$ inactivation in lung tumorigenesis. Our data strongly suggest that promoter methylation is the predominant mechanism involved in $b M L H 1$ and $b M S H 2$ alteration in NSCLC. More than 20 cases clearly showed retention of both alleles as well as aberrant methylation, indicating biallelic inactivation of $b M L H 1$ expression by promoter methylation. It is possible that these tumors belong to the so-called CpG island methylator phenotype group and have many genes whose expression is downregulated because of aberrant promoter methylation. Our published data on $p 16^{I N K 4 a}$ (34) and preliminary data on FHIT gene analyses showed that patients with aberrant promoter methylation in $b M L H 1$ and/or $b M S H 2$ often had hypermethylation at the promoter of multiple tumor suppressor genes. Although the mechanism of aberrant hypermethylation is unknown, it may be caused by increases in de novo methylation activity (35-37) or by a defect of the protection mechanism against de novo methylation (38). More study of candidate genes and genomic screening techniques that permit simultaneous analysis of promoter methylation of many genes should lead to a more complete knowledge of the epigenetic events occurring in tumors and their functional consequences.

\section{Acknowledgments}

This work was supported in part by grants NSC 90-2318-B-003-001 and NSC 91-3112-P-003-001 from the National Science Council, The Executive Yuan, Taipei, Taiwan, Republic of China.

\footnotetext{
1. Department of Health, The Executive Yuan, Republic of China. 2000. General Health Statistics, 1999. In Health and vital statistics, Republic of China. Republic of China Press. Taipei, Taiwan, Republic of China. 89-112.

2. Fishel, R., et al. 1993. The human mutator gene homolog MSH2 and its association with hereditary nonpolyposis colon cancer. Cell. 75:1027-1038.

3. Bronner, C.E., et al. 1994. Mutation in the DNA mismatch repair gene homologue hMLH1 is associated with hereditary non-polyposis colon cancer. Nature. 368:258-261.

4. Arzimanoglou, I.I., Gilbert, F., and Barber, H.R. 1998. Microsatellite instability in human solid tumors. Cancer. 82:1808-1820.

5. Kane, M.F., et al. 1997. Methylation of the hMLH1 promoter correlates with lack of expression of hMLH1 in sporadic colon tumors and mismatch repair-defective human tumor cell lines. Cancer Res. 57:808-811.

6. Cunningham, J.M., et al. 1998. Hypermethylation of the hMLH1 promoter in colon cancer with microsatellite instability. Cancer Res. 58:3455-3460.
} 
7. Leung, S.Y., et al. 1999. hMLH1 promoter methylation and lack of hMLH1 expression in sporadic gastric carcinomas with high-frequency microsatellite instability. Cancer Res. 59:159-164.

8. Esteller, M., et al. 1999. hMLH1 promoter hypermethylation is an early event in human endometrial tumorigenesis. Am. J. Pathol. 155:1767-1772.

9. Borresen, A.L., et al. 1995. Somatic mutations in the hMSH2 gene in microsatellite unstable colorectal carcinomas. Hum. Mol. Genet. 4:2065-2072.

10. Katabuchi, H., et al. 1995. Mutations in DNA mismatch repair genes are not responsible for microsatellite instability in most sporadic endometrial carcinomas. Cancer Res. 55:5556-5560.

11. Chang, J.W., et al. 2000. Correlation of genetic instability with mismatch repair protein expression and p53 mutations in non-small cell lung cancer. Clin. Cancer Res. 6:1639-1646.

12. Xinarianos, G., et al. 2000. hMLH1 and hMSH2 expression correlates with allelic imbalance on chromosome $3 p$ in non-small cell lung carcinomas. Cancer Res. 60:4216-4221.

13. Herman, J.G., Graff, J.R., Myohanen, S., Nelkin, B.D., and Baylin, S.B. 1996. Methylation-specific PCR: a novel PCR assay for methylation status of CpG islands. Proc. Natl. Acad. Sci. U. S. A. 93:9821-9826.

14. Herman, J.G., et al. 1998. Incidence and functional consequences of hMLH1 promoter hypermethylation in colorectal carcinoma. Proc. Natl. Acad. Sci.U. S. A. 95:6870-6875.

15. Nuovo, G.J., Plaia, T.W., Belinsky, S.A., Baylin, S.B., and Herman, J.G. 1999. In situ detection of the hypermethylation-induced inactivation of the 16 gene as an early event in oncogenesis. Proc. Natl. Acad. Sci. U. S. A 96:12754-12759.

16. Maekawa, M., et al. 1999. DNA methylation analysis using bisulfite treatment and PCR-single-strand conformation polymorphism in colorectal cancer showing microsatellite instability. Biochem. Biophys. Res. Commun. 262:671-676.

17. Deng, G., Chen, A., Hong, J., Chae, H.S., and Kim, Y.S. 1999. Methylation of $\mathrm{CpG}$ in a small region of the hMLH1 promoter invariably correlates with the absence of gene expression. Cancer Res. 59:2029-2033.

18. Ito, E., et al. 1999. A core promoter and a frequent single-nucleotide polymorphism of the mismatch repair gene hMLH1. Biochem. Biophys. Res. Commun. 256:488-494.

19. Belinsky, S.A., et al. 1998. Aberrant methylation of p16(INK4a) is an early event in lung cancer and a potential biomarker for early diagnosis. Proc. Natl. Acad. Sci. U. S. A. 95:11891-11896.

20. Zochbauer-Muller, S., et al. 2001. Aberrant promoter methylation of multiple genes in non-small cell lung cancers. Cancer Res. 61:249-255.

21. Palmisano, W.A., et al. 2000. Predicting lung cancer by detecting aberrant promoter methylation in sputum. Cancer Res. 60:5954-5958.

22. Mao, L., et al. 1994. Microsatellite alterations as clonal markers for the detection of human cancer. Proc. Natl. Acad. Sci. U. S. A. 91:9871-9875
23. Miozzo, M., et al. 1996. Microsatellite alterations in bronchial and sputum specimens of lung cancer patients. Cancer Res. 56:2285-2288.

24. Ahrendt, S.A., et al. 1999. Molecular detection of tumor cells in bronchoalveolar lavage fluid from patients with early stage lung cancer. J. Natl. Cancer Inst. 91:332-339.

25. Ger, L.P., Hsu, W.L., Chen, K.T., and Chen, C.J. 1993. Risk factors of lung cancer by histological category in Taiwan. Anticancer Res. 13:1491-1500.

26. Esteller, M., Corn, P.G., Baylin, S.B., and Herman, J.G. 2001. A gene hypermethylation profile of human cancer. Cancer Res. 61:3225-3229.

27. Aubry, M.C., et al. 2001. DNA mismatch repair genes $h M L H 1, h M S H 2$, and $\mathrm{bMSH} 6$ are not inactivated in bronchioloalveolar carcinomas of the lung. Cancer. 92:2898-2901.

28. Liu, Y.Y., Chen, Y.M., Huang, M.H., and Perng, R.P. 2000. Prognosis and recurrent patterns in bronchioloalveolar carcinoma. Chest. 118:940-947.

29. Benachenhou, N., Guiral, S., Gorska-Flipot, I., Labuda, D., and Sinnett, D. 1998. High resolution deletion mapping reveals frequent allelic losses at the DNA mismatch repair loci hMLH1 and hMSH3 in non-small cell lung cancer. Int. J. Cancer. 77:173-180.

30. Zienolddiny, S., Ryberg, D., Arab, M.O., Skaug, V., and Haugen, A. 2001. Loss of heterozygosity is related to p53 mutations and smoking in lung cancer. Br. J. Cancer. 84:226-231.

31. Wistuba, I.I., et al. 2000. High resolution chromosome 3p allelotyping of human lung cancer and preneoplastic/preinvasive bronchial epithelium reveals multiple, discontinuous sites of $3 p$ allele loss and three regions of frequent breakpoints. Cancer Res. 60:1949-1960.

32. Hirao, T., et al. 2001. Tobacco smoke-induced DNA damage and an early age of smoking initiation induce chromosome loss at 3 p 21 in lung cancer. Cancer Res. 61:612-615.

33. Roche, J., et al. 1996. Distinct 3p21.3 deletions in lung cancer and identification of a new human semaphorin. Oncogene. 12:1289-1297.

34. Chen, J.T., Chen, Y.C., Wang, Y.C., Chen, C.Y., and Wang, Y.C. 2002. Alterations of the $p 16^{I N K 4 a}$ gene in resected non-small cell lung tumors and exfoliated cells within sputum. Int. J. Cancer. 98:724-731.

35. Belinsky, S.A., Nikula, K.J., Baylin, S.B., and Issa, J.P. 1996. Increased cytosine DNA-methyltransferase activity is target-cell-specific and an early event in lung cancer. Proc. Natl. Acad. Sci. U. S. A. 93:4045-4050.

36. Kanai, Y., Ushijima, S., Kondo, Y., Nakanishi, Y., and Hirohashi, S. 2001. DNA methyltransferase expression and DNA methylation of CPG islands and peri-centromeric satellite regions in human colorectal and stomach cancers. Int. J. Cancer. 91:205-212.

37. Mizuno, S., et al. 2001. Expression of DNA methyltransferases DNMT1, $3 \mathrm{~A}$, and $3 \mathrm{~B}$ in normal hematopoiesis and in acute and chronic myelogenous leukemia. Blood. 97:1172-1179.

38. Graff, J.R., Herman, J.G., Myohanen, S., Baylin, S.B., and Vertino, P.M. 1997. Mapping patterns of CpG island methylation in normal and neoplastic cells implicates both upstream and downstream regions in de novo methylation. J. Biol. Chem. 272:22322-22329. 